\title{
Epigenetic changes within the promoter region of the HLA-G gene in ovarian tumors
}

\author{
Laura Menendez ${ }^{1}$, L DeEtte Walker ${ }^{2,3}$, Lilya V Matyunina ${ }^{2,3}$, \\ Kimberly A Totten ${ }^{2,3}$, Benedict B Benigno ${ }^{3}$ and John F McDonald*2,3
}

\begin{abstract}
Address: ${ }^{1}$ Department of Genetics, University of Georgia, Athens, GA 30605, USA, 25chool of Biology, Georgia Institute of Technology, Atlanta GA 30332, USA and ${ }^{3}$ Ovarian Cancer Institute, Atlanta, GA 30342, USA

Email: Laura Menendez - Imenen@uga.edu; L DeEtte Walker - dwalker@gatech.edu; Lilya V Matyunina - lilya.matyunina@biology.gatech.edu; Kimberly A Totten - kt92@mail.gatech.edu; Benedict B Benigno - bbenigno@seqynonc.com; John F McDonald* - mcgene@gatech.edu

* Corresponding author
\end{abstract}

Published: 22 May 2008

Molecular Cancer 2008, 7:43 doi:10.1186/1476-4598-7-43
Received: II March 2008

Accepted: 22 May 2008

This article is available from: http://www.molecular-cancer.com/content/7/1/43

(C) 2008 Menendez et al; licensee BioMed Central Ltd.

This is an Open Access article distributed under the terms of the Creative Commons Attribution License (http://creativecommons.org/licenses/by/2.0), which permits unrestricted use, distribution, and reproduction in any medium, provided the original work is properly cited.

\begin{abstract}
Background: Previous findings have suggested that epigenetic-mediated HLA-G expression in tumor cells may be associated with resistance to host immunosurveillance. To explore the potential role of DNA methylation on HLA-G expression in ovarian cancer, we correlated differences in HLA-G expression with methylation changes within the HLA-G regulatory region in an ovarian cancer cell line treated with 5-aza-deoxycytidine (5-aza-dC) and in malignant and benign ovarian tumor samples and ovarian surface epithelial cells (OSE) isolated from patients with normal ovaries.
\end{abstract}

Results: A region containing an intact hypoxia response element (HRE) remained completely methylated in the cell line after treatment with 5 -aza- $\mathrm{dC}$ and was completely methylated in all of the ovarian tumor (malignant and benign) samples examined, but only variably methylated in normal OSE samples. HLA-G expression was significantly increased in the 5 -aza-dC treated cell line but no significant difference was detected between the tumor and OSE samples examined.

Conclusion: Since HRE is the binding site of a known repressor of HLA-G expression (HIF-I), we hypothesize that methylation of the region surrounding the HRE may help maintain the potential for expression of HLA-G in ovarian tumors. The fact that no correlation exists between methylation and HLA-G gene expression between ovarian tumor samples and OSE, suggests that changes in methylation may be necessary but not sufficient for HLA-G expression in ovarian cancer.

\section{Background}

Classic and non-classic HLA (human leukocyte antigen) class I genes play a central role in the regulation of the immune response. The non-classic HLA-G gene is expressed in a variety of tissues but perhaps most notably in the fetal-maternal interface on the extravillous cytotrophoblast and has been postulated to help protect the fetus from maternal allorecognition [1]. This hypothesis is supported by subsequent studies demonstrating that $H L A-G$ proteins can suppress a variety of immune functions including natural killer (NK) cell-mediated cytolysis and the T-cell proliferative response [2,3]. Recent findings indicate that HLA-G antigens are present in ovarian and various other types of malignant cells and tissues [4-7]. 
These findings and others have led to the hypothesis that induction of HLA-G expression in tumor cells may contribute to their avoidance of immunosurveillance by the host $[8,9]$ (but disputed by [10]).

Sequences known to be involved in the transcriptional regulation of most HLA class I genes are disrupted in the HLA-G gene raising questions as to the mechanisms underlying HLA-G expression [11-13]. Studies conducted in a variety of human cancer cell lines suggest that epigenetic mechanisms may play an important role in HLA-G expression $[14,15]$. To explore the potential role of DNA methylation on HLA-G expression in ovarian cancer, we tested the effect of the methylation inhibitor 5-aza-deoxycytidine on methylation within the CpG-enriched regulatory region of the HLA-G gene and correlated changes in expression in an ovarian cancer cell line. The results demonstrate that 5-aza-dC treatment results in hypomethylation of putative control sequences within the 5 ' regulatory region of HLA-G and that these changes in methylation correlate with a significant increase in expression. A notable exception was a region (-211 to -290) containing a hypoxia response element (HRE; [16]) that remained completely methylated.

Methylation within the regulatory region of the HLA-G gene also was examined in eighteen malignant and benign ovarian tumor samples and in ovarian surface epithelial cells (OSE) isolated from four patients with normal ovaries. A number of significant differences in levels of methylation of sequences within the 5 ' regulatory region were detected between the tumor samples and the normal surface epithelial cells. Interestingly, the region containing the HRE (-211 to -290) that remained methylated in 5-aza-dC treated BG-1 cells was also completely methylated in all ovarian tumor samples, but not in OSE controls, suggesting strong selection against accessibility to the HRE in ovarian tumor cells. Although the highest levels of HLA-G expression were associated with tumor samples, no significant overall correlation between methylation and expression levels was detected by real time RT-PCR. Our results indicate that alterations in methylation may be necessary but not sufficient for HLA$G$ expression in ovarian tumors.

\section{Results}

5-aza-dC treatment of ovarian cancer cells (BG-I) results in hypomethylation of sequences within the HLA-G regulatory region and correlates with an increase in gene expression

Previous studies have shown that 5-aza-deoxycytidine treatment of a variety of tumor (glioma, choriocarcinoma, B-lymphoma and melanoma) cell lines results in significant hypomethylation of a CpG-rich region located within 450 bp $5^{\prime}$ of the HLA-G start codon and correlates with a significant increase in HLA-G expression [15]. To determine if ovarian tumor cells would show a similar response, we selected an ovarian cancer cell line (BG-1) that did not display HLA-G expression prior to treatment (Figure 1). Sodium bisulfite modification and subsequent sequencing was carried out on 10 clones each of genomic DNA isolated from 5-aza-dC treated and untreated BG-1 cells. The results demonstrate that while all $19 \mathrm{CpG}$ sites located within the 450 bp region $5^{\prime}$ of the HLA-G start codon were methylated in untreated cells, there was a $36 \%$ overall decrease of methylation in treated cells (Figure 2). The approximately 200 bp region immediately $5^{\prime}$ to the transcriptional start site $(-8$ to -188$)$ that encompasses HLA-G cis-regulatory sequences was significantly ( $\mathrm{p}$ $=0.001)$ hypomethylated, but a $79 \mathrm{bp}$ region $(-211$ to 290) remained methylated after 5 -aza-dC treatment in all of the clones examined. Correlated with the 5-aza-dC induced changes in methylation patterns, there was an increase in HLA-G expression (Figure 1).

\section{Methylation within the HLA-G 5 ' regulatory region significantly differs between ovarian surface epithelial cells (OSEs) and benign and malignant ovarian tumors}

Epithelial ovarian carcinomas are believed to derive from ovarian surface epithelial cells (OSE) or epithelial cells lining the inner surface of inclusion cysts $[17,18]$. To determine if methylation within the 5 ' regulatory region of the HLA-G gene differs among OSE and benign and malignant ovarian tumors, we performed sodium bisulfite genomic sequencing of DNA isolated from laser capture microdissected (LCM) tumor cells from 9 adenomas and 9 adenocarcinomas and OSE brushings from the

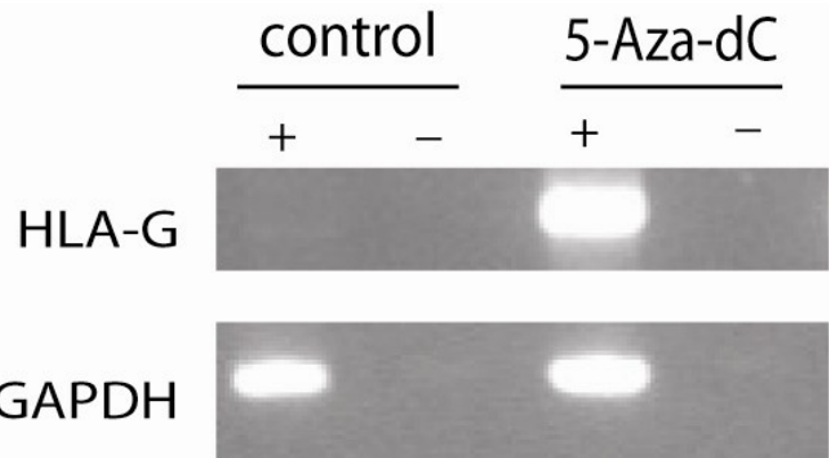

Figure I

Activation of HLA-G gene transcription in the BG-I cell line treated with 5-aza-deoxycytidine (5-aza-dC). Results of semi-quantitative RT-PCR (reverese transcriptasepolymerase chain reaction) on the ovarian carcinoma cell line BG-I, either untreated (control) or treated with $50 \mu \mathrm{M} 5$ aza-deoxycytidine (5-aza-dC). ("-" = samples without RT; "+" = samples with RT). GAPDH was used as an endogenous control. 
(A)

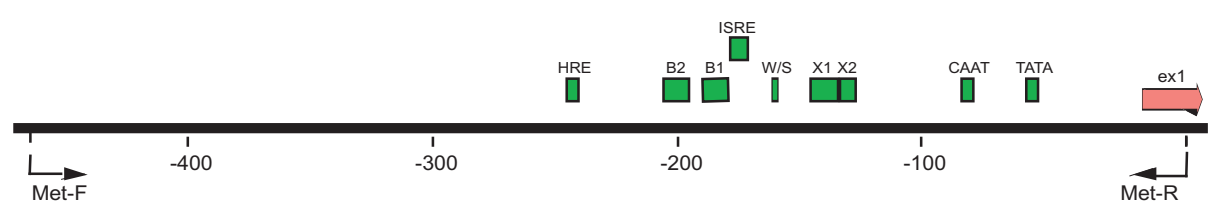

(B)
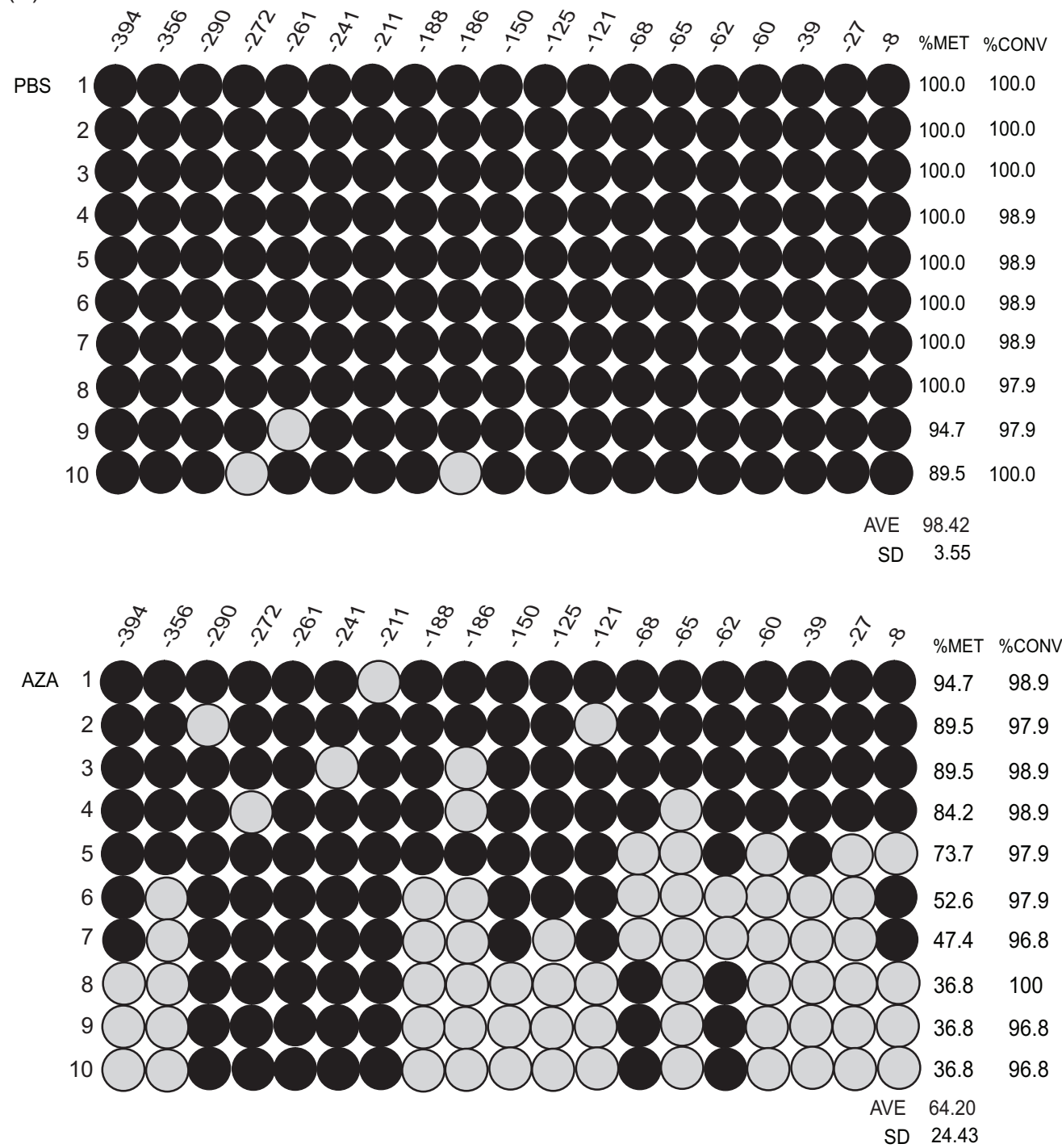

Figure 2

Methylation analysis of the HLA-G promoter in the control and 5-aza-dC treated BG-I cell line. (A) Schematic map of 450 bp of the HLA-G promoter region. Colored boxes represent enhancers and regulator binding sites: $\mathrm{HRE}=$ hypoxia response element; $\mathrm{B} 2$ = enhancer $\kappa \mathrm{B} 2 ; \mathrm{BI}=$ enhancer $\kappa \mathrm{BI}$; ISRE = interferon sequence responsive element; W/S= W/S box; $X I=$ conserved $X I$ regulatory box; $X 2=X 2$ box; $C A A T=$ CCAAT box; TATA = TATA box; exI = exon I; Met-F and Met-R $=$ forward $(F)$ and reverse $(R)$ primer binding sites. (B) Bisulfite genomic sequencing of 19 CpG dinucleotides of the region from -450 to ATG. Individual CpG dinulcotides are depicted as circles. Each row of circles represents an individual sequenced clone, either untreated (PBS) or treated with $50 \mu \mathrm{M} 5$-aza-dC (open circle $=100 \%$ unmethylated; filled circle $=100 \%$ methylated; \%MET = percentage of methylation of each individual clone; \%CONV = efficiency of sodium bisulfite treatment). 
Table I: Methylation status of $19 \mathrm{CpG}$ sites in the 450 bp promoter region of HLA-G gene in malignant (CA), benign (BN) and normal (NL) ovarian tissue samples.

\begin{tabular}{|c|c|c|c|c|c|c|c|c|c|c|c|c|c|c|c|c|c|c|c|}
\hline $\mathrm{CA}$ & -394 & -356 & -290 & -272 & -261 & -241 & -211 & -188 & -186 & -150 & -125 & -121 & -68 & -65 & -62 & -60 & -39 & -27 & -8 \\
\hline 212 & 0 & 0 & 1 & 1 & 1 & 1 & 1 & 0.00 & 0.00 & 0.25 & 0.00 & 0.25 & 0.75 & 0.00 & 0.75 & 0.00 & 0.00 & 0.00 & 0.75 \\
\hline 229 & 0 & 0 & 1 & 1 & 1 & I & I & 0.00 & 0.00 & 1.00 & 0.00 & 1.00 & 0.00 & 0.00 & 0.00 & 0.00 & 0.00 & 0.00 & 0.00 \\
\hline 183 & 0 & 0 & 1 & 1 & 1 & I & 1 & 0.00 & 0.00 & 0.38 & 0.00 & 0.38 & 0.63 & 0.00 & 0.63 & 0.00 & 0.00 & 0.00 & 0.63 \\
\hline 317 & 0 & 0 & 1 & 1 & 1 & 1 & 1 & 0.60 & 0.60 & 0.80 & 0.00 & 0.80 & 0.20 & 0.20 & 0.80 & 0.00 & 0.60 & 0.00 & 0.40 \\
\hline 369 & 0 & 0 & 1 & 1 & 1 & I & 1 & 0.40 & 0.40 & 0.60 & 0.20 & 0.60 & 0.40 & 0.00 & 1.00 & 0.20 & 0.60 & 0.00 & 0.20 \\
\hline 413 & 0 & 0 & 1 & 1 & 1 & 1 & 1 & 0.60 & 0.60 & 1.00 & 0.00 & 0.80 & 0.00 & 0.00 & 0.80 & 0.00 & 0.60 & 0.00 & 0.20 \\
\hline 170 & 0 & 0 & I & I & I & I & 1 & 0.20 & 0.20 & 1.00 & 0.00 & 1.00 & 0.00 & 0.00 & 0.20 & 0.00 & 0.20 & 0.00 & 0.00 \\
\hline 228 & 0 & 0 & 1 & 1 & 1 & 1 & 1 & 0.75 & 0.75 & 0.75 & 0.00 & 0.75 & 0.25 & 0.25 & 1.00 & 0.00 & 0.75 & 0.00 & 0.25 \\
\hline 242 & 0 & 0 & I & I & 1 & 1 & 1 & 0.20 & 0.20 & 0.40 & 0.00 & 0.40 & 0.60 & 0.00 & 0.80 & 0.00 & 0.20 & 0.00 & 0.80 \\
\hline AVE & 0.000 & 0.000 & 1.000 & 1.000 & 1.000 & 1.000 & 1.000 & 0.306 & 0.306 & 0.687 & 0.022 & 0.664 & 0.314 & 0.050 & 0.664 & 0.022 & 0.328 & 0.000 & 0.359 \\
\hline \multicolumn{20}{|l|}{ BN } \\
\hline 382 & 0 & 0 & I & I & 1 & 1 & 1 & 0.00 & 0.00 & 0.40 & 0.00 & 0.40 & 0.60 & 0.00 & 0.60 & 0.00 & 0.00 & 0.00 & 0.80 \\
\hline 412 & 0 & 0 & 1 & 1 & 1 & 1 & 1 & 0.60 & 0.80 & 0.80 & 0.00 & 0.80 & 0.20 & 0.00 & 0.83 & 0.00 & 0.60 & 0.00 & 0.00 \\
\hline 388 & 0 & 0 & I & I & I & 1 & 1 & 0.17 & 0.17 & 0.17 & 0.00 & 0.17 & 0.67 & 0.00 & 1.00 & 0.00 & 0.33 & 0.00 & 0.83 \\
\hline 416 & 0.20 & 0 & I & I & 1 & 1 & I & 0.40 & 0.40 & 0.60 & 0.00 & 0.60 & 0.40 & 0.00 & 0.80 & 0.00 & 0.40 & 0.00 & 0.60 \\
\hline 371 & 0.14 & 0 & 1 & 1 & 1 & 1 & $I$ & 0.14 & 0.14 & 0.43 & 0.00 & 0.43 & 0.57 & 0.00 & 0.71 & 0.00 & 0.29 & 0.00 & 0.71 \\
\hline 377 & 0.20 & 0.20 & 0.60 & 1 & 1 & 1 & 1 & 0.40 & 0.60 & 0.60 & 0.20 & 0.60 & 0.20 & 0.00 & 0.60 & 0.00 & 0.60 & 0.00 & 0.00 \\
\hline 386 & 0.20 & 0 & 1 & I & I & I & I & 0.00 & 0.00 & 0.60 & 0.00 & 0.60 & 0.40 & 0.00 & 0.40 & 0.00 & 0.00 & 0.00 & 0.40 \\
\hline 400 & 0 & 0 & 1 & 1 & 1 & 1 & 1 & 0.20 & 0.50 & 0.50 & 0.00 & 0.50 & 0.50 & 0.00 & 1.00 & 0.00 & 0.50 & 0.00 & 0.50 \\
\hline 383 & 0 & 0 & 1 & 1 & 1 & 1 & 1 & 0.10 & 0.10 & 0.90 & 0.00 & 0.90 & 0.10 & 0.00 & 0.30 & 0.00 & 0.10 & 0.00 & 0.13 \\
\hline AVE & 0.082 & 0.022 & 0.956 & 1.000 & 1.000 & 1.000 & 1.000 & 0.223 & 0.301 & 0.556 & 0.022 & 0.556 & 0.404 & 0.000 & 0.694 & 0.000 & 0.313 & 0.000 & 0.441 \\
\hline \multicolumn{20}{|l|}{$\mathrm{NL}$} \\
\hline 492 & 0.40 & 0.40 & 0.40 & 0.60 & 0.80 & 1.00 & 0.80 & 0.00 & 0.20 & 0.20 & 0.20 & 0.20 & 0.40 & 1.00 & 0.20 & 0.20 & 0.00 & 0.00 & 0.20 \\
\hline 499 & 0.17 & 0.33 & 0.50 & 0.67 & 0.83 & 0.67 & 0.83 & 0.50 & 0.00 & 0.33 & 0.50 & 0.50 & 0.50 & 0.50 & 0.67 & 0.17 & 0.00 & 0.00 & 0.17 \\
\hline 500 & 0.60 & 0.80 & 0.00 & 0.80 & 0.40 & 0.80 & 0.80 & 0.00 & 0.00 & 0.20 & 0.20 & 0.20 & 0.00 & 0.00 & 0.00 & 0.00 & 0.20 & 0.00 & 0.00 \\
\hline 525 & 0.33 & 0.33 & 0.00 & 1.00 & 1.00 & 1.00 & 0.67 & 0.00 & 0.00 & 0.00 & 0.33 & 0.33 & 0.33 & 0.33 & 0.67 & 0.00 & 0.00 & 0.33 & 0.33 \\
\hline AVE & 0.374 & 0.466 & 0.225 & 0.767 & 0.758 & 0.867 & 0.776 & 0.125 & 0.050 & 0.183 & 0.308 & 0.308 & 0.308 & 0.458 & 0.384 & 0.092 & 0.050 & 0.083 & 0.174 \\
\hline
\end{tabular}

P-VALUES

$\begin{array}{lllllllllllllllllllll}\text { CA VS NL } & <I E-04 & <I E-04 & <I E-04 & 0.001 & 0.011 & 0.025 & <I E-04 & 0.308 & 0.122 & 0.008 & 0.0004 & 0.033 & 0.970 & 0.014 & 0.225 & 0.174 & 0.111 & 0.139 & 0.277\end{array}$

$\begin{array}{llllllllllllllllllll}B N & & \end{array}$

Values shown for each sample/position are the average of 5-8 clones. Significance of differences at each CpG site between tumor and normal samples are shown.

normal ovaries of 4 patients undergoing hysterectomies for reasons unrelated to ovarian cancer. Five to eight clones were sequenced from each DNA sample. The average \% methylation for all $19 \mathrm{CpG}$ sites located from 8 to 394 bp $5^{\prime}$ to the HLA-G transcriptional start site is presented in Table 1 and Figure 3.

Average methylation levels across the 450 bp 5' region examined were variable among samples, ranging from $26.3 \%$ to $55.3 \%$. The malignant and benign tumor samples displayed nearly identical average methylation levels across the region (adenocarcinomas 45.9\%; adenomas $45.1 \%$ ) while average methylation levels (35.5\%) were significantly lower for the OSE samples (adenocarcinoma vs. OSE, $\mathrm{p}=0.022$; adenoma vs. OSE, $\mathrm{p}=0.007$, unpaired t-test). The same region (CpG sites -211 to -290 ) that remained methylated after 5-aza-dC treatment in the BG1 cell line was uniformly hypermethylated in all of the tumor samples, but variably methylated in the OSE samples.

Examination of the methylation levels between the benign and malignant tumor samples at each of the 19 individual $\mathrm{CpG}$ sites displayed no significant differences (Table 1). However, 12 of the 19 sites displayed significant differences between the OSE and tumor samples (Table 1). The OSE samples displayed significantly higher levels of methylation than tumor samples at 5 (-60, -65, $125,-356$ and -394 ) of the 19 CpG sites examined. OSE samples displayed significantly lower levels of methylation than tumor samples at $7(-121,-150,-211,-241$, 261, -272, and -290) of the 19 CpG sites examined.

We also examined a second 399 bp region of the HLA-G CpG island from -7 to +392 extending into Exon 2. This region contains $42 \mathrm{CpG}$ dinucleotides. No significant dif- 
(A)

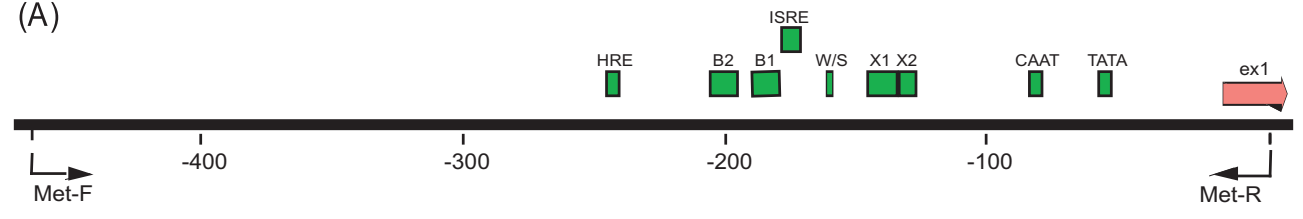

(B)
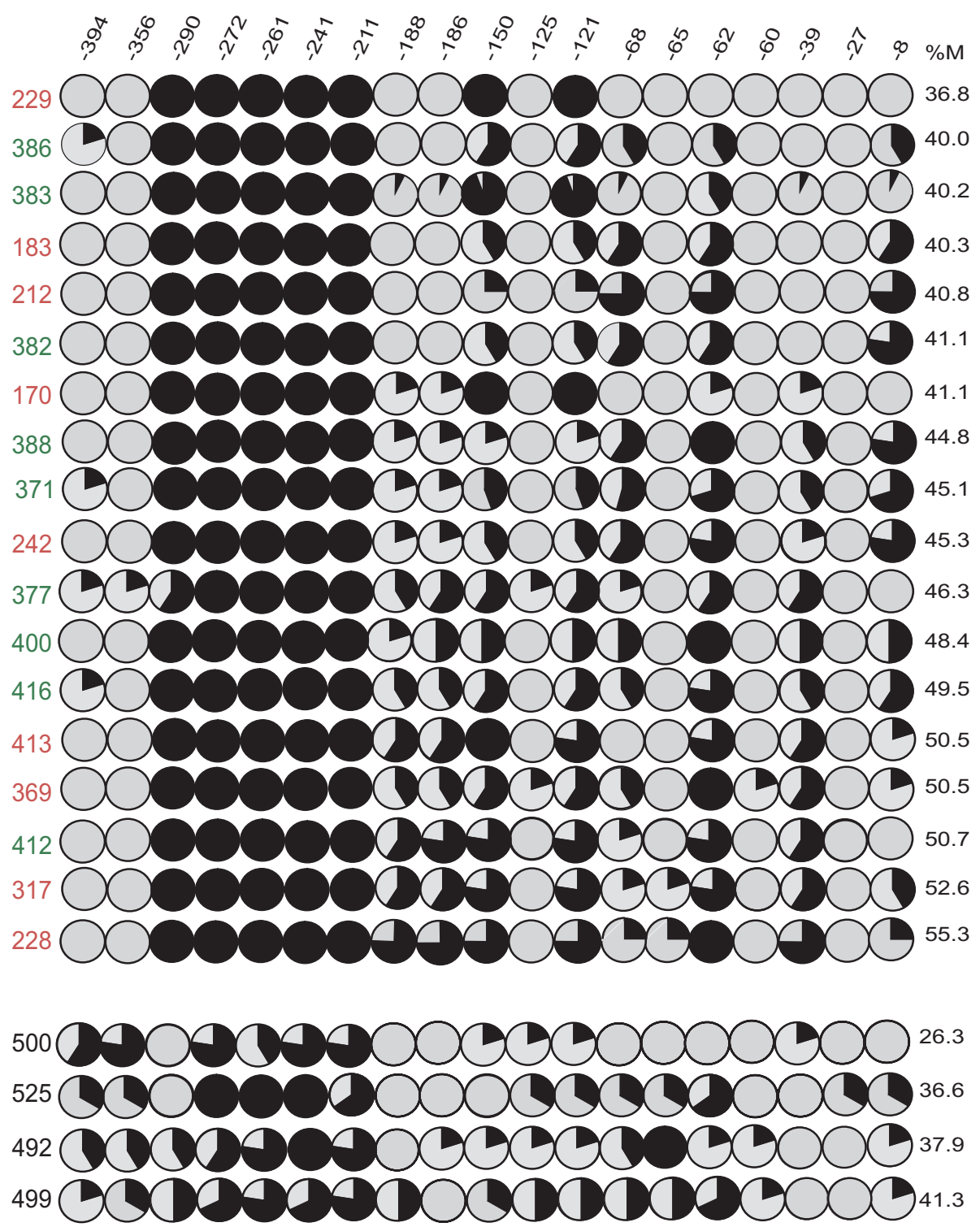

Figure 3

Methylation analysis of the HLA-G promoter in tumor and normal ovarian samples. (A) Schematic map of 450 bp of the HLA-G promoter region (See Figure 2 legend for symbols). (B) Bisulfite genomic sequencing of 19 CpG dinucleotides of the region from -450 to the transcription start site is analyzed for 18 ovarian adenocarcinomas (red) and benign adenomas (green) and four normal ovaries (black). Five to eight clones were sequenced for each sample and each circle represents the average methylation for a single $\mathrm{CPG}$ dinucleotide (open circle $=100 \%$ unmethylated; filled circle $=100 \%$ methylated; $\% \mathrm{M}=$ percentage of total methylation per sample). 
Table 2: Methylation status of 42 CpG sites in a 399 bp region of HLA-G gene extending from the transcriptional start site (TSS) into Exon II $(-7$ to +392$)$ in malignant $(C A)$, benign $(B N)$ and normal $(N L)$ ovarian tissue samples.

\begin{tabular}{|c|c|c|c|c|c|c|c|c|c|c|c|c|c|c|c|c|c|}
\hline$C A$ & +18 & +40 & +59 & +70 & +80 & +97 & +106 & +108 & +124 & +129 & +132 & +138 & +147 & +154 & +156 & +159 & $+|7|$ \\
\hline 212 & 0.63 & 0.50 & 0.50 & 0.50 & 0.50 & 0.75 & 0.63 & 0.63 & 0.50 & 0.00 & 0.50 & 0.50 & 0.50 & 0.50 & 0.50 & 0.50 & 0.50 \\
\hline 183 & 0.50 & 0.50 & 0.50 & 0.50 & 0.50 & 0.00 & 0.50 & 0.50 & 0.50 & 0.00 & 0.50 & 0.50 & 0.50 & 0.50 & 0.50 & 0.50 & 0.50 \\
\hline 369 & 0.80 & 0.90 & 0.90 & 0.80 & 0.80 & 0.80 & 0.80 & 0.80 & 0.58 & 0.00 & 0.80 & 0.70 & 0.68 & 0.80 & 0.68 & 0.80 & 0.68 \\
\hline 242 & 0.38 & 0.39 & 0.45 & 0.39 & 0.32 & 0.25 & 0.13 & 0.32 & 0.32 & 0.25 & 0.32 & 0.32 & 0.32 & 0.32 & 0.32 & 0.32 & 0.32 \\
\hline 170 & 1.00 & 0.60 & 1.00 & 1.00 & 1.00 & 1.00 & 1.00 & 1.00 & 0.88 & 0.00 & 1.00 & 1.00 & 1.00 & 1.00 & 1.00 & 1.00 & 1.00 \\
\hline 413 & 1.00 & 1.00 & 1.00 & 1.00 & 0.93 & 0.50 & 1.00 & 0.88 & 1.00 & 0.00 & 1.00 & 1.00 & 1.00 & 0.88 & 1.00 & 1.00 & 1.00 \\
\hline 317 & 0.88 & 0.88 & 0.88 & 0.88 & 0.88 & 0.50 & 0.88 & 0.88 & 0.88 & 0.00 & 0.88 & 0.88 & 0.88 & 0.88 & 0.88 & 0.88 & 0.88 \\
\hline 228 & 1.00 & 1.00 & 1.00 & 1.00 & 1.00 & 1.00 & 1.00 & 1.00 & 1.00 & 0.00 & 0.50 & 1.00 & 1.00 & 1.00 & 1.00 & 1.00 & 1.00 \\
\hline AVE & 0.77 & 0.72 & 0.78 & 0.76 & 0.74 & 0.60 & 0.74 & 0.75 & 0.71 & 0.03 & 0.69 & 0.74 & 0.73 & 0.73 & 0.73 & 0.75 & 0.73 \\
\hline
\end{tabular}

$\mathrm{BN}$

\begin{tabular}{lllllllllllllllllll}
\hline 382 & 0.58 & 0.71 & 0.58 & 0.58 & 0.46 & 0.50 & 0.58 & 0.58 & 0.73 & 0.00 & 0.58 & 0.58 & 0.58 & 0.58 & 0.58 & 0.58 & 0.58 \\
412 & 1.00 & 1.00 & 1.00 & 1.00 & 1.00 & 0.75 & 1.00 & 1.00 & 0.75 & 0.13 & 0.63 & 0.75 & 1.00 & 1.00 & 1.00 & 1.00 & 1.00 \\
388 & 1.00 & 1.00 & 1.00 & 1.00 & 1.00 & 0.50 & 1.00 & 0.63 & 1.00 & 0.00 & 1.00 & 1.00 & 1.00 & 1.00 & 1.00 & 1.00 & 1.00 \\
416 & 0.50 & 0.67 & 0.50 & 0.50 & 0.50 & 0.50 & 0.50 & 0.50 & 0.50 & 0.00 & 0.50 & 0.50 & 0.17 & 0.50 & 0.50 & 0.50 & 0.50 \\
371 & 1.00 & 1.00 & 1.00 & 1.00 & 1.00 & 1.00 & 1.00 & 1.00 & 0.83 & 0.17 & 1.00 & 1.00 & 1.00 & 1.00 & 1.00 & 1.00 & 1.00 \\
377 & 1.00 & 1.00 & 1.00 & 1.00 & 1.00 & 0.38 & 1.00 & 0.88 & 0.75 & 0.17 & 0.88 & 0.88 & 0.88 & 1.00 & 1.00 & 0.88 & 1.00 \\
386 & 1.00 & 1.00 & 1.00 & 1.00 & 0.88 & 0.88 & 1.00 & 1.00 & 0.71 & 0.50 & 0.83 & 0.83 & 0.83 & 1.00 & 1.00 & 1.00 & 1.00 \\
400 & 1.00 & 1.00 & 1.00 & 1.00 & 1.00 & 1.00 & 0.67 & 0.67 & 0.70 & 0.00 & 0.50 & 1.00 & 1.00 & 1.00 & 1.00 & 1.00 & 1.00 \\
383 & 1.00 & 1.00 & 1.00 & 1.00 & 1.00 & 0.00 & 1.00 & 1.00 & 1.00 & 0.00 & 0.00 & 1.00 & 1.00 & 1.00 & 1.00 & 1.00 & 1.00 \\
& & & & & & & & & & & & & & & & & & \\
AVE & $\mathbf{0 . 9 0}$ & $\mathbf{0 . 9 3}$ & $\mathbf{0 . 9 0}$ & $\mathbf{0 . 9 0}$ & $\mathbf{0 . 8 7}$ & $\mathbf{0 . 6 1}$ & $\mathbf{0 . 8 6}$ & $\mathbf{0 . 8 1}$ & $\mathbf{0 . 7 8}$ & $\mathbf{0 . 1 1}$ & $\mathbf{0 . 6 6}$ & $\mathbf{0 . 8 4}$ & $\mathbf{0 . 8 3}$ & $\mathbf{0 . 9 0}$ & $\mathbf{0 . 9 0}$ & $\mathbf{0 . 8 8}$ & $\mathbf{0 . 9 0}$
\end{tabular}

NL

\begin{tabular}{|c|c|c|c|c|c|c|c|c|c|c|c|c|c|c|c|c|c|}
\hline 500 & 1.00 & 0.83 & 0.83 & 0.83 & 1.00 & 0.67 & 0.83 & 0.83 & 1.00 & 0.00 & 0.83 & 0.83 & 0.50 & 0.50 & 0.33 & 0.50 & 0.50 \\
\hline 525 & 0.75 & 0.75 & 0.63 & 0.75 & 0.75 & 0.50 & 0.75 & 0.88 & 0.75 & 0.00 & 0.75 & 0.75 & 0.75 & 0.75 & 0.75 & 0.75 & 0.63 \\
\hline 492 & 0.63 & 0.50 & 0.50 & 0.50 & 0.50 & 0.25 & 0.63 & 0.63 & 0.63 & 0.00 & 0.63 & 0.50 & 0.63 & 0.63 & 0.63 & 0.63 & 0.75 \\
\hline 499 & 0.75 & 0.71 & 0.58 & 0.71 & 0.58 & 0.29 & 0.88 & 0.88 & 0.54 & 0.00 & 0.71 & 0.71 & 0.88 & 0.58 & 0.71 & 0.71 & 0.88 \\
\hline AVE & 0.78 & 0.70 & 0.64 & 0.70 & 0.71 & 0.43 & 0.77 & 0.80 & 0.73 & 0.00 & 0.73 & 0.70 & 0.69 & 0.61 & 0.60 & 0.65 & 0.69 \\
\hline
\end{tabular}

P-VALUES

\begin{tabular}{|c|c|c|c|c|c|c|c|c|c|c|c|c|c|c|c|c|c|}
\hline CA VS NL & 0.946 & 0.869 & 0.322 & 0.673 & 0.838 & 0.390 & 0.857 & 0.690 & 0.879 & 0.506 & 0.766 & 0.795 & 0.762 & 0.403 & 0.415 & 0.487 & 0.762 \\
\hline BN VS NL & 0.332 & 0.018 & 0.041 & 0.105 & 0.255 & 0.320 & 0.446 & 0.990 & 0.660 & 0.235 & 0.671 & 0.220 & 0.380 & 0.025 & 0.032 & 0.051 & 0.10 \\
\hline CA & +175 & +193 & +227 & +230 & +233 & +240 & +245 & +249 & +251 & +261 & +269 & $+28 I$ & +287 & +292 & +299 & +303 & +308 \\
\hline 212 & 0.50 & 0.50 & 0.50 & 0.50 & 0.50 & 0.50 & 0.50 & 0.38 & 0.00 & 0.50 & 0.50 & 0.50 & 0.50 & 0.50 & 0.38 & 0.50 & 0.50 \\
\hline 183 & 0.50 & 0.50 & 0.50 & 0.50 & 0.50 & 0.50 & 0.50 & 0.50 & 0.50 & 0.50 & 0.50 & 0.50 & 0.50 & 0.50 & 0.50 & 0.50 & 0.50 \\
\hline 369 & 0.58 & 0.68 & 0.80 & 0.80 & 0.80 & 0.80 & 0.80 & 0.90 & 0.80 & 0.70 & 0.80 & 0.80 & 0.80 & 0.80 & 0.80 & 0.80 & 0.68 \\
\hline 242 & 0.32 & 0.32 & 0.46 & 0.39 & 0.39 & 0.39 & 0.32 & 0.32 & 0.32 & 0.39 & 0.46 & 0.32 & 0.32 & 0.32 & 0.20 & 0.32 & 0.39 \\
\hline 170 & 1.00 & 1.00 & 1.00 & 1.00 & 1.00 & 1.00 & 1.00 & 1.00 & 1.00 & 0.63 & 1.00 & 1.00 & 1.00 & 1.00 & 1.00 & 1.00 & 1.00 \\
\hline 413 & 1.00 & 1.00 & 0.88 & 0.88 & 0.88 & 0.88 & 0.88 & 0.75 & 0.88 & 0.75 & 0.88 & 0.88 & 0.63 & 1.00 & 1.00 & 1.00 & 1.00 \\
\hline 317 & 0.88 & 0.38 & 1.00 & 1.00 & 1.00 & 1.00 & 1.00 & 1.00 & 1.00 & 1.00 & 1.00 & 1.00 & 1.00 & 0.50 & 1.00 & 1.00 & 1.00 \\
\hline 228 & 1.00 & 1.00 & 1.00 & 1.00 & 1.00 & 1.00 & 1.00 & 1.00 & 1.00 & 1.00 & 1.00 & 1.00 & 1.00 & 1.00 & 1.00 & 1.00 & 1.00 \\
\hline
\end{tabular}

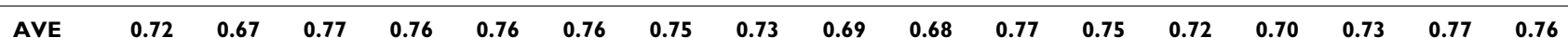

$\mathrm{BN}$

$\begin{array}{lllllllllllllllllll}382 & 0.58 & 0.58 & 0.58 & 0.58 & 0.58 & 0.58 & 0.58 & 0.58 & 0.46 & 0.58 & 0.71 & 0.58 & 0.46 & 0.58 & 0.58 & 0.58 & 0.58 \\ 412 & 0.63 & 1.00 & 1.00 & 1.00 & 1.00 & 1.00 & 1.00 & 1.00 & 1.00 & 1.00 & 1.00 & 1.00 & 1.00 & 1.00 & 1.00 & 1.00 & 1.00 \\ 388 & 1.00 & 1.00 & 1.00 & 1.00 & 1.00 & 1.00 & 1.00 & 1.00 & 1.00 & 0.75 & 1.00 & 1.00 & 1.00 & 1.00 & 1.00 & 1.00 & 1.00 \\ 416 & 0.50 & 0.50 & 0.50 & 0.50 & 0.50 & 0.50 & 0.50 & 0.50 & 0.50 & 0.50 & 0.50 & 0.50 & 0.50 & 0.50 & 0.50 & 0.50 & 0.67 \\ 371 & 1.00 & 1.00 & 1.00 & 1.00 & 1.00 & 1.00 & 1.00 & 1.00 & 1.00 & 0.17 & 1.00 & 1.00 & 1.00 & 1.00 & 1.00 & 1.00 & 1.00 \\ 377 & 1.00 & 0.88 & 1.00 & 1.00 & 1.00 & 1.00 & 1.00 & 0.88 & 1.00 & 0.88 & 1.00 & 1.00 & 0.88 & 1.00 & 1.00 & 1.00 & 1.00 \\ 386 & 1.00 & 1.00 & 1.00 & 1.00 & 1.00 & 1.00 & 1.00 & 1.00 & 1.00 & 0.83 & 1.00 & 1.00 & 1.00 & 1.00 & 1.00 & 1.00 & 0.88\end{array}$


Table 2: Methylation status of 42 CpG sites in a 399 bp region of HLA-G gene extending from the transcriptional start site (TSS) into Exon II (-7 to +392) in malignant (CA), benign (BN) and normal (NL) ovarian tissue samples. (Continued)

\begin{tabular}{|c|c|c|c|c|c|c|c|c|c|c|c|c|c|c|c|c|c|}
\hline 400 & 1.00 & 1.00 & 1.00 & 1.00 & 1.00 & 0.70 & 1.00 & 1.00 & 1.00 & 1.00 & 1.00 & 1.00 & 1.00 & 1.00 & 1.00 & 1.00 & 1.00 \\
\hline 383 & 1.00 & 1.00 & 1.00 & 1.00 & 1.00 & 1.00 & 1.00 & 1.00 & 1.00 & 1.00 & 1.00 & 1.00 & 1.00 & 1.00 & 1.00 & 1.00 & 1.00 \\
\hline AVE & 0.86 & 0.88 & 0.90 & 0.90 & 0.90 & 0.86 & 0.90 & 0.88 & 0.88 & 0.75 & 0.91 & 0.90 & 0.87 & 0.90 & 0.90 & 0.90 & 0.90 \\
\hline \multicolumn{18}{|l|}{$\mathrm{NL}$} \\
\hline 500 & 0.50 & 0.50 & 0.50 & 0.50 & 0.50 & 0.50 & 0.50 & 0.50 & 0.50 & 0.50 & 0.50 & 0.50 & 0.83 & 0.50 & 0.50 & 0.50 & 0.50 \\
\hline 525 & 0.75 & 0.63 & 0.75 & 0.75 & 0.75 & 0.75 & 0.75 & 0.75 & 0.75 & 0.75 & 0.75 & 0.88 & 0.63 & 0.63 & 0.75 & 0.75 & 0.75 \\
\hline 492 & 0.75 & 0.88 & 0.75 & 0.75 & 0.63 & 0.75 & 0.75 & 0.75 & 0.75 & 0.75 & 0.75 & 0.75 & 0.75 & 0.75 & 0.63 & 0.75 & 0.63 \\
\hline 499 & 0.88 & 0.88 & 0.88 & 0.88 & 0.88 & 0.88 & 0.88 & 0.88 & 0.88 & 0.88 & 0.88 & 0.88 & 0.88 & 0.88 & 0.88 & 0.88 & 0.88 \\
\hline AVE & 0.72 & 0.72 & 0.72 & 0.72 & 0.69 & 0.72 & 0.72 & 0.72 & 0.72 & 0.72 & 0.72 & 0.75 & 0.77 & 0.69 & 0.69 & 0.72 & 0.69 \\
\hline \multicolumn{18}{|l|}{ P-VALUES } \\
\hline CA VS NL & 0.986 & 0.776 & 0.725 & 0.784 & 0.640 & 0.784 & 0.840 & 0.941 & 0.877 & 0.788 & 0.725 & 0.998 & 0.720 & 0.923 & 0.799 & 0.780 & 0.650 \\
\hline BN VS NL & 0.283 & 0.200 & 0.148 & 0.148 & 0.096 & 0.241 & 0.148 & 0.173 & 0.222 & 0.865 & 0.096 & 0.250 & 0.427 & 0.100 & 0.100 & 0.148 & 0.053 \\
\hline $\mathrm{CA}$ & +314 & +319 & +322 & +328 & +340 & +342 & +346 & +349 & & & & & & & & & \\
\hline 212 & 0.50 & 0.50 & 0.50 & 0.50 & 0.50 & 0.50 & 0.50 & 0.50 & & & & & & & & & \\
\hline 183 & 0.50 & 0.50 & 0.50 & 0.50 & 0.50 & 0.50 & 0.50 & 0.50 & & & & & & & & & \\
\hline 369 & 0.80 & 0.80 & 0.80 & 0.80 & 0.80 & 0.80 & 0.80 & 0.80 & & & & & & & & & \\
\hline 242 & 0.32 & 0.39 & 0.32 & 0.45 & 0.45 & 0.45 & 0.45 & 0.45 & & & & & & & & & \\
\hline 170 & 1.00 & 1.00 & 1.00 & 1.00 & 1.00 & 1.00 & 1.00 & 1.00 & & & & & & & & & \\
\hline 413 & 1.00 & 1.00 & 1.00 & 1.00 & 1.00 & 1.00 & 1.00 & 1.00 & & & & & & & & & \\
\hline 317 & 1.00 & 1.00 & 1.00 & 1.00 & 1.00 & 1.00 & 1.00 & 1.00 & & & & & & & & & \\
\hline 228 & 1.00 & 1.00 & 1.00 & 1.00 & 1.00 & 1.00 & 1.00 & 1.00 & & & & & & & & & \\
\hline AVE & 0.77 & 0.77 & 0.77 & 0.78 & 0.78 & 0.78 & 0.78 & 0.78 & & & & & & & & & \\
\hline \multicolumn{18}{|l|}{$\mathrm{BN}$} \\
\hline 382 & 0.58 & 0.58 & 0.58 & 0.58 & 0.58 & 0.58 & 0.58 & 0.58 & & & & & & & & & \\
\hline 412 & 1.00 & 1.00 & 1.00 & 1.00 & 1.00 & 1.00 & 1.00 & 1.00 & & & & & & & & & \\
\hline 388 & 1.00 & 1.00 & 1.00 & 1.00 & 1.00 & 1.00 & 1.00 & 1.00 & & & & & & & & & \\
\hline 416 & 0.50 & 0.50 & 0.50 & 0.50 & 0.50 & 0.50 & 0.50 & 0.50 & & & & & & & & & \\
\hline 371 & 1.00 & 1.00 & 1.00 & 1.00 & 1.00 & 1.00 & 1.00 & 1.00 & & & & & & & & & \\
\hline 377 & 1.00 & 1.00 & 1.00 & 1.00 & 1.00 & 1.00 & 1.00 & 1.00 & & & & & & & & & \\
\hline 386 & 1.00 & 1.00 & 1.00 & 1.00 & 1.00 & 1.00 & 1.00 & 1.00 & & & & & & & & & \\
\hline 400 & 1.00 & 1.00 & 0.83 & 1.00 & 1.00 & 1.00 & 1.00 & 1.00 & & & & & & & & & \\
\hline 383 & 1.00 & 1.00 & 1.00 & 1.00 & 1.00 & 1.00 & 1.00 & 1.00 & & & & & & & & & \\
\hline AVE & 0.90 & 0.90 & 0.88 & 0.90 & 0.90 & 0.90 & 0.90 & 0.90 & & & & & & & & & \\
\hline \multicolumn{18}{|l|}{$\mathrm{NL}$} \\
\hline 500 & 0.50 & 0.50 & 0.50 & 0.33 & 0.50 & 0.50 & 0.50 & 0.50 & & & & & & & & & \\
\hline 525 & 0.75 & 0.75 & 0.75 & 0.75 & 0.75 & 0.75 & 0.75 & 0.75 & & & & & & & & & \\
\hline 492 & 0.75 & 0.75 & 0.75 & 0.75 & 0.75 & 0.75 & 0.75 & 1.00 & & & & & & & & & \\
\hline 499 & 0.88 & 0.88 & 0.88 & 0.88 & 0.88 & 0.88 & 0.88 & 0.88 & & & & & & & & & \\
\hline AVE & 0.72 & 0.72 & 0.72 & 0.68 & 0.72 & 0.72 & 0.72 & 0.78 & & & & & & & & & \\
\hline
\end{tabular}

P-VALUES

$\begin{array}{lllllllll}\text { CA VS NL } & 0.769 & 0.714 & 0.769 & 0.515 & 0.67 \mid & 0.67 \mid & 0.67 \mid & 0.998\end{array}$

$\begin{array}{lllllllll}\text { BN VS NL } & 0.148 & 0.148 & 0.190 & 0.120 & 0.148 & 0.148 & 0.148 & 0.365\end{array}$

Values shown for each sample/position are the average of 5-8 clones. Marginally significant differences between tumor and normal samples were detected at 4 of the 42 CpG sites. 
ference overall in levels of methylation between OSE and malignant or benign ovarian tumor samples was detected within this region $(\mathrm{p}<0.05$; Table 2$)$.

\section{No significant difference in HLA-G expression was detected between OSE and malignant and benign ovarian tumor samples}

To determine if levels of HLA-G expression are significantly different between ovarian tumors and OSE cells, we determined relative expression levels (real time RT-PCR) of 3 OSE samples and tumor cells isolated by LCM from 4 malignant and 4 benign ovarian tumor samples. The results presented in Table 3 indicate that HLA-G expression levels are highly variable among all samples and no consistent or significant differences in HLA-G expression were detected between the OSE and tumor samples. Our results are consistent with previous reports of high variability in HLA-G expression within and between tumors $[7,10]$. Thus, the significant difference in methylation levels within the promoter region observed between OSE and the ovarian tumor samples was not correlated with differences in HLA-G expression.

\section{Discussion}

Contrary to other HLA molecules, HLA-G regulation is only partially understood. Studies of the methylation levels of $450 \mathrm{bp}$ of the promoter region of HLA-G in melanoma and choriocarcinoma cell lines showed a correlation between expression and low levels of methylation [15]. In our study we looked at the same region in the ovarian cancer cell line BG-1, which showed almost complete methylation of all CpG dinucleotides and no expression of HLA-G. Treatment of the cells with the methylation inhibitor, 5-aza-dC, resulted in a significant decrease in levels of methylation at the HLA-G promoter region that correlated with an increase in levels of $H L A-G$ expression (Figures 1 and 2). Consistent with previously published studies conducted with other cancer cell lines $[14,15]$, our results suggest that the methylation status of the HLA-G promoter region may be important for the control of HLA-G expression in ovarian cancer.
To test this hypothesis in tissues, we analyzed methylation of the HLA-G 5 ' regulatory region in 4 OSE samples and 18 malignant and benign ovarian tumor samples. In addition, we measured the relative levels of $H L A-G$ expression in 11 OSE and ovarian tumor samples from which cancer cells were isolated using laser capture microdissection (LCM). We found that there were significant differences in levels of methylation within the 5' HLA-G regulatory region between OSE and the malignant and benign ovarian tumor samples, but no significant difference between the malignant and benign tumor samples. This observation is consistent with the view that any putative adaptive advantage imparted to tumor cells by HLA-G apparently does not distinguish between malignant and benign tumor cells.

We found that many of the CpG sites displaying a significant decrease in methylation in the tumor samples were located in proximity to intact binding sites of regulatory proteins known to serve as activators of gene expression (CCAAT box -76 and an intact SP1 and RFX binding site within the X1 box at $-130[13,19]$; Figure 3$)$. Hypomethylation of these sites in ovarian tumors may serve to potentiate HLA-G expression. In contrast, we observed a significant increase in levels of methylation in tumor samples at CpG sites located in proximity to a hypoxia response element [16] located 243 bp upstream of the $H L A-G$ transcriptional start site. Interestingly, this same region remained methylated in 5-aza-dC treated BG-1 cells, suggesting that strong selection may operate to prevent binding to the HRE in ovarian tumor cells.

HRE is the binding site of HIF-1, a protein that plays a critical role in the cellular response to hypoxia, with the potential to act as either a positive or negative regulatory factor [20-22]. Evidence has recently been reported that HIF-1 may act as a negative regulator of HLA-G expression in some human melanoma (FON) and choriocarcinoma (JEG-3) cell lines [22] The functional significance of the methylation of the CpG island surrounding the HIF-1 binding site in the ovarian tumor samples remains to be determined. However, preventing the binding of a poten-

Table 3: Real-time RT-PCR analysis of HLA-G expression in ovarian samples.

\begin{tabular}{|c|c|c|c|c|c|c|}
\hline \multicolumn{3}{|c|}{ ADENOCARCINOMAS } & \multicolumn{2}{|c|}{ ADENOMAS } & \multicolumn{2}{|c|}{ NORMAL } \\
\hline Patient & Expression & FIGO Stage & Patient & Expression & Patient & Expression \\
\hline 242 & 0.42 & IIIc & 386 & 0.67 & 500 & 3.50 \\
\hline 369 & 3.05 & IIlc & 371 & 0.83 & 492 & 20.53 \\
\hline 183 & 4.03 & IIlc & 416 & 3.54 & 525 & 53.71 \\
\hline 212 & 177.01 & Illc & 382 & 13.36 & & \\
\hline AVE & 46.13 & & AVE & 4.60 & AVE & 25.91 \\
\hline SD & 87.27 & & SD & 5.98 & SD & 25.53 \\
\hline
\end{tabular}


tial repressor protein (HIF-1) may help maintain the transcription of HLA-G in ovarian tumors under hypoxic conditions thus allowing the tumor cells to evade cytotoxic T lymphocyte recognition and destruction.

It is important to keep in mind that the postulated significance of methylation changes in gene regulatory regions is the increased (hypomethylation) or decreased (hypermethylation) access it provides for the binding of proteins or protein complexes that regulate gene expression [23]. Thus, although methylation changes in gene regulatory regions may be necessary for subsequent changes in levels of expression, these changes in methylation alone may not be sufficient to effect expression changes. The fact that the significant differences in methylation we observed between OSE and ovarian tumor samples do not correlate with differences in HLA-G expression may serve to underlie this distinction. Our results are consistent with a scenario whereby changes in methylation in ovarian tumor cells potentiate them for HLA-G transcriptional activation (or avoidance of transcriptional repression) by regulatory proteins induced by micro-environmental or mutational changes in specific tumor cell lineages.

\section{Conclusion}

Differences in methylation within the 5 ' regulatory region of HLA-G were detected between normal ovarian surface epithelial cells and ovarian cancers. The most striking difference was in a region (-211 to -290 from HLA-G TSS), containing an intact hypoxia response element (HRE). This region was completely methylated in all of the ovarian tumor (malignant and benign) samples examined, but was only variably methylated in normal surface epithelial cells. Interestingly, this same region remained completely methylated in an ovarian cancer cell line (BG-1) after treatment with 5 -aza-dC suggesting that there is strong selection against loss of methylation in this region in these ovarian cancer cells. Since HRE is the binding site of a known repressor of HLA-G expression (HIF-1), we hypothesize that methylation of the region surrounding the HRE may help maintain the potential for expression of HLA-G in ovarian tumors. The fact that no correlation exists between methylation and HLA-G gene expression between ovarian tumor samples and OSE, leads us to conclude that changes in methylation may be necessary but not sufficient for HLA-G expression in ovarian cancer.

\section{Methods}

\section{Tissue samples}

Ovarian tumor samples were collected at Northside Hospital (Atlanta, GA) during surgery and snap frozen in liquid nitrogen within 1 minute of removal from patients. Brushings of normal ovarian surface epithelial cells (OSE) were preserved in RNAlater (Ambion, Austin, GA) within 1 minute of removal from patients. Patient consent and approval from the Institutional Review Boards of the University of Georgia, Georgia Institute of Technology, and Northside Hospital were obtained. Pathological and clinical information of samples is presented in Table 4.

\section{Human cell-line culture}

The BG-1 ovarian adenocarcinoma cell line was kindly provided by JM Hall and KS Korach (NIH). Cells were propagated in DMEM: F12/50:50 (Invitrogen, Carlsbad, $\mathrm{CA}$ ), supplemented with $2 \mathrm{mM}$ L-glutamine (Mediatech Inc., Herndon, VA), 10\% heat inactivated FBS (Invitrogen, Carlsbad, CA), $1 \mathrm{mM}$ sodium pyruvate (Mediatech Inc., Herndon, VA), and $1 \%$ penicillin and streptomycin (Mediatech, Inc., Herndon, VA) at $37^{\circ} \mathrm{C}, 5 \% \mathrm{CO}^{2}$ and $\sim 80 \%$ relative humidity.

\section{Cell treatments}

BG-1 cells were plated at low density 16 hours before treatment with $50 \mu \mathrm{M}$ 5-aza-deoxycytidine (Sigma, St. Louis, MO) for 5 days. On the third day of treatment, the media was replaced with fresh media containing 5-azadC. Control cells treated with PBS were grown for the same amount of time.

\section{Laser capture microdissection (LCM)}

Fresh frozen tissues from tumors were cut into sevenmicron sections, applied to non-charged slides, then fixed in $75 \%$ ethanol for 30 seconds, stained and dehydrated using the HistoGene LCM Frozen Section Staining Kit (Arcturus, Mountain View, CA). LCM was performed with an AutoPix Automated Laser Capture Microdissection System using the CapSure Macro Caps (Arcturus, Mountain View, CA). Approximately 10,000 cells were captured on each of 3-4 caps per sample. Subsequently the DNA was extracted from captured cells using the PicoPure DNA Extraction Kit (Arcturus, Mountain View, CA). RNA extraction from captured cells was carried out using the PicoPure RNA Extraction Kit and amplified with the RiboAmpHS RNA Amplification Kit (Arcturus, Mountain View, CA).

\section{DNA and RNA extraction from ovarian surface epithelial cells}

Epithelial brushings were obtained from normal ovaries and kept in RNAlater (Qiagen, Valencia, CA). For DNA extraction, cells were spun down and re-suspended in lysis buffer (10 mM Tris HCl, $10 \mathrm{mM}$ EDTA, 0.2\% SDS and 50 $\mathrm{mM} \mathrm{NaCl}$ ), with $50 \mu \mathrm{g} / \mathrm{ml}$ RNAse A (Invitrogen, Carlsbad, CA) and $100 \mu \mathrm{g} / \mathrm{ml}$ Proteinase K (EM Science, Gibbstown, $\mathrm{NJ}$ ) overnight at $37^{\circ} \mathrm{C}$. DNA was then phenol-extracted and ethanol precipitated. For RNA extraction, cells were spun down and Trizol was added, following the manufacturer's recommended protocol (Invitrogen, Carlsbad, CA). cDNA was synthesized from RNA using the Ribo- 
Table 4: Patient samples analyzed in this study

\begin{tabular}{|c|c|c|c|c|}
\hline Patient & Histology & Age & FIGO Stage & Grade \\
\hline 228 & Clear cell adenocarcinoma & 55 & la & N/A \\
\hline 317 & Serous papillary adenocarcinoma & 59 & lc & 3 \\
\hline 170 & Mixed endometrioid/serous papillary adenocarcinoma & 57 & Ila & 2 \\
\hline 413 & Serous papillary adenocarcinoma & 50 & Ilb & 3 \\
\hline 212 & Mixed endometrioid/serous papillary adenocarcinoma & 59 & Illc & 3 \\
\hline 369 & Serous papillary adenocarcinoma & 52 & Illc & 3 \\
\hline 242 & Serous papillary adenocarcinoma & 64 & Illc & 3 \\
\hline 229 & Serous papillary adenocarcinoma & 58 & IIlc & 3 \\
\hline 183 & Serous papillary adenocarcinoma & 66 & IIlc & 2 \\
\hline 412 & Mucinous cystadenoma & 65 & & \\
\hline 377 & Mucinous cystadenoma & 42 & & \\
\hline 383 & Mucinous cystadenoma with focal borderline features & 75 & & \\
\hline 382 & Serous cystadenofibroma & 67 & & \\
\hline 386 & Serous cystadenofibroma & 59 & & \\
\hline 388 & Serous cystadenofibroma & 53 & & \\
\hline 371 & Serous cystadenofibroma & 60 & & \\
\hline 400 & Serous cystadenofibroma & 43 & & \\
\hline 416 & Serous cystadenoma & 65 & & \\
\hline 492 & Normal surface epithelium & 38 & & \\
\hline 499 & Normal surface epithelium & 69 & & \\
\hline 500 & Normal surface epithelium & 25 & & \\
\hline 525 & Normal surface epithelium & 70 & & \\
\hline
\end{tabular}

AmpHS RNA Amplification Kit (Arcturus, Mountain View, CA).

\section{RT-PCR}

\section{Semi-quantitative}

Total RNA was extracted from BG-1 cells by adding $1 \mathrm{ml}$ of Trizol (Invitrogen, Carlsbad, CA) according to the manufacturer's recommendations, and further purified using RNEasy Mini Kit (Qiagen, Valencia, CA). Four to six $\mu \mathrm{g}$ of DNAse-treated RNA (DNA-free; Ambion, Houston, TX) were used to generate cDNA by oligo-dT reverse transcription with the Superscript RT-PCR Kit (Invitrogen, Carlsbad, CA). Primers that recognize all HLA-G isoforms were designed from the HLA-G RNA reference sequence (X12273) at 257F 5'-GGAAGAGGAGACACGGAACA-3' and 1200R 5'-TCCTGTTCCAGAAAAGGGG-3'. GAPDH (NM_002046) was used as an endogenous control, using primers 287F 5'-GAAATCCCATCACCATCTTCCAG-3' and 599R 5'-ATGAGTCCTTCCACGATACAAAAG-3'.

\section{Real-time $R T$-PCR}

Total RNA extracted from LCM captured ovarian tumor cells and normal OSE cell was converted to amplified cDNA for real-time RT-PCR. TaqMan Gene Expression Assays (Applied Biosystems, Foster City, CA) were conducted following manufacturer's protocol for HLA-G (Hs00365950_g1) and for GAPDH endogenous control (Hs99999905_m1) using the DNA Engine Opticon System (MJ Research, Waltham, MA). A standard curve of serial dilutions of a plasmid containing either HLA-G or
GAPDH was established for each assay to obtain a relative quantification of HLA-G or GAPDH gene expression. For each sample normalized $H L A-G$ expression values are presented as the ratio between the target gene (HLA-G) and the endogenous control (GAPDH).

\section{Sodium bisulfite genomic sequencing}

Five hundred nanograms to one microgram of genomic DNA from the BG-1 cells, LCM captured ovarian tumor cells and normal OSE cells were modified with sodium bisulfite according to Herman et al. [24]. For LCM samples, sodium bisulfite modification was performed by pooling DNA from 2 to 3 caps per patient sample, and adding $15 \mu \mathrm{l}$ of hydroquinone and $200 \mu \mathrm{l}$ of sodium bisulfite, as well as $1 \mu \mathrm{g}$ of Herring Sperm DNA (Promega, Madison, WI) as a carrier.

Modified DNA was used for PCR with primers for the CpG-enriched 5' regulatory region of HLA-G [15]. Position of the primers from the ATG codon of the HLA-G gene are: Primer F (-456) 5'-AAGAGTATAGGAGGATAGGTAAGG-3' and primer R (-16) 5'-AACACCATAACCACCATCCTTAAC-3'.

A second region of $H L A-G$ from -7 to +392 from the ATG codon was amplified performing two rounds of PCR. The first round was done with primers F-5'-GGATTTATTTTTTTTAGATGTTAAG-3' and R-5'-ATCTACAAATTCATTCTATCAATCTATAC-3', and then nested PCR was performed with F-5'-GTTAAGGATGGTGGTTATGGTGTT- 
3' and R-5'-TATCTCCTCTTCCCAATACTCCAA-3'. PCR products were purified using the QIAquick Gel Extraction Kit (Qiagen, Valencia, CA), and the DNA cloned using TOPO TA Cloning for Sequencing Kit (Invitrogen, Carlsbad, CA). DNA plasmids from individual colonies were extracted using QIAprep Spin Miniprep Kit (Qiagen, Valencia, CA). Automated sequencing was performed by Integrated Biotech Laboratories (Athens, GA).

\section{Competing interests}

The authors declare that they have no competing interests.

\section{Authors' contributions}

LM, JM Conceived and designed the experiments, LM, LDW, LVM Performed the experiments, LM, JFM, LDW Analyzed the data, KAT, BB Contributed reagents/materials, LM, JFM, LDW Drafted the manuscript:. All authors read and approved the final manuscript.

\section{Acknowledgements}

This research was supported by the Deborah Nash Harris Foundation, the Robinson Family Foundation, the Golfers Against Cancer Foundation, the Ovarian Cycle Foundation and the Larry and Beth Lawrence Foundation.

\section{References}

I. Kovats S, Main EK, Librach C, Stubblebine M, Fisher SJ, DeMars R: A class I antigen, HLA-G, expressed in human trophoblasts. Science 1990, 248:220-223.

2. Carosella ED, Moreau P, Le Maoult J, Le Discorde M, Dausset J, Rouas-Freiss N: HLA-G molecules: from maternal-fetal tolerance to tissue acceptance. Adv Immunol 2003, 8I:199-252.

3. Lin A, Yan W-H, Xu H-H, Gan M-F, Cai J-F, Zhu M, Zhou M-Y: HLAG expression in human ovarian carcinoma counteracts NK cell function. Ann Oncol 2007, I 8(I I): 1804-I809.

4. Lefebvre S, Antoine M, Uzan S, McMaster M, Dausset J, Carosella ED, Paul P: Specific activation of the non-classical class I histocompatibility HLA-G antigen and expression of the ILT2 inhibitory receptor in human breast cancer. J Pathol 2002, 196:266-274.

5. Bukur J, Malenica B, Huber C, Seliger B: Altered expression of nonclassical HLA class Ib antigens in human renal cell carcinoma and its association with impaired immune response. Hum Immunol 2003, 64:1081-1092.

6. Rouas-Freiss N, Moreau P, Ferrone S, Carosella D: HLA-G proteins in cancer: do they provide tumor cells with an escape mech anism? Cancer Res 2005, 65:10139-10144.

7. Sheu JJ-C, Shih IM: Clinical and biological significance of HLAG expression in ovarian cancer. Semin Cancer Biol 2007, I 7(6):436-443.

8. Paul P, Rouas-Freiss N, Khalil-Daher I, Moreau P, Riteau B, Le Gal FA Avril MF, Dausset J, Guillet JG, Carosella ED: HLA-G expression in melanoma: a way for tumor cells to escape from immunosurveillance. Proc Natl Acad Sci USA 1998, 95:45I 0-45I5.

9. Rouas-Freiss N, Moreau P, Menier C, LeMaoult J, Carosella D: Expression of tolerogenic HLA-G molecules in cancer prevents antitumor responses. Semin Cancer Biol 2007, I 7(6):4|3-42I.

10. Real LM, Cabrera T, Collado A, Jimenez P, Garcia A, Ruiz-Cabello F, Garrido F: Expression of HLA G in human tumors is not a frequent event. Int J Cancer 1999, 81:512-518.

II. Gobin SJ, Keijsers V, Cheong C, van Zutphen M, Elsen PJ Van den Transcriptional regulation of HLA-G. Transplant Proc 1999, 31:1857-|859.

12. Solier C, Mallet V, Lenfant F, Bertrand A, Hucheng A, Le Bouteiller P. HLA-G unique promoter region: functional implications. Immunogenetics 200I, 53:617-625.

13. Tripathi P, Agrawal S: Non-classical HLA-G antigen and its role in the cancer progression. Cancer lnvest 2006, 24:178-186.
14. Moreau P, Mouillot G, Rousseau P, Marcou C, Dausset J, Carosella ED: HLA-G gene repression is reversed by demethylation. Proc Natl Acad Sci USA 2003, 100: I 191-1 I 96.

15. Mouillot G, Marcou C, Rousseau P, Rouas-Freiss N, Carosella ED, Moreau P: HLA-G gene activation in tumor cells involves cisacting epigenetic changes. Int / Cancer 2005, I I 3:928-936.

16. Chang $C-C$, Ferrone S: HLA-G in melanoma: can the current controversies be solved? Semin Cancer Biol 2003, I3(5):36I-369.

17. Auersperg N, Wong AST, Choi K-C, Kang SK, Leung PCK: Ovarian surface epithelium: Biology, endocrinology, and pathology. Endocr Rev 200I, 22(2):255-288.

18. Shih I-M, Kurman RJ: Ovarian tumorigenesis: a proposed model based on morphological and molecular genetic analysis. Amer J Path 2004, 164:15|I-1518.

19. Rousseau P, Paul P, O'Brien M, Dausset J, Carosella ED, Moreau P. The XI box of HLA-G promoter is a target for RFX and Sp I factors. Human Immunol 2000, 6 I: I I32-I I 37.

20. Manalo DJ, rowan A, Lavoie T, Natarajan L, Kelly BD, Ye SQ, Garcia JGN, Semenza GL: Transcriptional regulation of vascular endothelial cell responses to hypoxia by HIF-I. Blood 2005, 105:659-669.

21. Eltzchig HK, Abdulla P, Hoffman E, Hamilton KE, Daniels D, et al: HIF-I-dependent repression of equilibrative nucleoside transporter (ENT) in hypoxia. J Exp Med 2005, 202:I493-I505.

22. Mouillot G, Marcou C, Zidi I, Guillard C, Sangrouber D, Carosella ED, Moreau P: Hypoxia modulates HLA-G gene expression in tumor cells. Hum Immunol 2007, 68(4):277-285.

23. D'Alessio AC, Szyf M: Epigenetic têtê-à-têtê: the bilateral relationship between chromatin modifications and DNA methylation. Biochem Cell Biol 2006, 84:463-476.

24. Herman JG, Graff JR, Myohanen S, Nelkin BD, Baylin SB: Methylation-specific PCR: a novel PCR assay for methylation status of CpG islands. Proc Natl Acad Sci USA 1 996, 93:982I-9826.

Publish with BioMed Central and every scientist can read your work free of charge

"BioMed Central will be the most significant development for disseminating the results of biomedical research in our lifetime. "

Sir Paul Nurse, Cancer Research UK

Your research papers will be:

- available free of charge to the entire biomedical community

- peer reviewed and published immediately upon acceptance

- cited in PubMed and archived on PubMed Central

-yours - you keep the copyright 